\title{
Exérese de Schwanoma por meio da técnica de hemilaminectomia dorsolateral cervical cranial
}

\author{
Schwanoma removal using the technique of cranial cervical dorsolateral hemilaminectomy
}

Juliana Voll', Rodolfo Voll'², Leandro Gaiga', Cristiano Gomes' \& Márcio Poletto Ferreira'

\begin{abstract}
RESUMO
Os tumores intradurais-extramedulares representam 50\% das neoplasias do sistema nervoso. A maioria dos tumores intradurais-extramedulares caninos corresponde a neoplasias de raízes nervosas (neurofibromas, neurofibrossarcomas, schwanomas) e meningiomas. Optamos por descrever um caso de schwanoma uma vez que não o são rotineiramente relatados. Este trabalho refere-se a um cão da raça Pastor Belga, macho, 8 anos, atendido no Serviço de Neurologia do Hospital de Clínicas Veterinárias da Faculdade de Veterinária da Universidade Federal do Rio Grande do Sul com histórico de déficit de reação postural e hemiparesia dos membros torácico e pélvico do lado direito. $\mathrm{O}$ exame mielográfico evidenciou existência de massa subdural-extramedular no lado direito junto à articulação atlanto-occipital, compatível com neoplasia. $\mathrm{O}$ cão foi submetido a tratamento cirúrgico utilizando-se hemilaminectomia dorsolateral cervical cranial e remoção parcial do material compressivo. Setenta e cinco dias após a cirurgia o animal apresentou sinais de piora. O exame mielográfico evidenciou aumento de volume do tumor em relação ao inicial. Foi realizada exérese do tumor, porém, ao término do procedimento cirúrgico o paciente foi a óbito; essa situação condiz com a literatura, onde apenas uma pequena porcentagem desses tumores é completamente removida e as recidivas são altas.
\end{abstract}

Descritores: schwanoma, hemilaminectomia dorsolateral cervical cranial, tumores intradurais-extramedulares.

\section{ABSTRACT}

Intradural-extramedular tumors represent $50 \%$ of nervous system neoplasias. The majority of canine intradural-extramedular tumors corresponds to nervous roots neoplasias (neurofibromas, neurofibrossarcomas, schwanomas) and meningiomas. We opt for describing a schwanoma case once they are not seen routinely. This report mentions a Belgian Sheep dog, male, 8 years old, taken care of in Neurology Service of the Clinical Veterinarians Hospital of Veterinary Medicine College of Federal University of Rio Grande do Sul presenting deficit or postural reaction and hemiparesis of the right side thoracic and pelvic limbs. Mielographic examination evidenced subdural-extramedular mass existence at the right side near by the atlanto-occipital joint, compatible with neoplasia. The dog was submitted to surgical treatment using cranial cervical dorsolateral laminectomy and removal of compressive substance. Seventy-five days after the surgery the animal presented worsening signals, clinical and neurological examination had evidenced enlargement of tumor volume in comparison to the initial one. It was done partial exeresis of the tumor, however by the ending of surgical procedure the patient perished. This situation matches with literature, where only a small percentage of these tumors are completely removed and reappearance rates are high.

Key words: schwanoma, cranial cervical dorsolateral laminectomy, intradural-extramedular tumors. 


\section{INTRODUÇÃO}

Os tumores podem surgir a partir de muitos locais no e ao redor do cordão espinhal [5,6]. O diagnóstico diferencial do tipo de tumores vertebrais ou do cordão espinhal se baseia na localização do tumor (osso, cordão espinhal, raiz nervosa ou metástase) e na classificação mielográfica (extradural, intraduralextramedular ou intramedular) [6].

Os Schwanomas são tumores intraduraisextramedulares com origem nas bainhas dos nervos (nas células de schawn dos axônios) [2,9] com comportamento habitualmente benigno [6,7] de crescimento lento $[4,10]$. Esses tumores acometem geralmente cães com mais de cinco anos de idade $[4,6,13]$, não apresentando predileção racial, no entanto, machos são um pouco mais predispostos $(1,4: 1)$ [12]. Comumente são localizados na região cervical em cães $[1,10,13]$.

$\mathrm{O}$ tratamento de escolha em casos de schwanoma consiste na ressecção cirúrgica $[4,8,12]$ ou na redução em massa dos tumores de raízes nervosas [1], onde a hemilaminectomia $[1,10,12,13]$, durotomia e remoção do tumor são necessárias em casos de envolvimento de raízes nervosas [1]. Os objetivos cirúrgicos incluem a exposição do tumor, a ressecção ampla do tecido neoplásico, e a descompressão do cordão espinhal e raiz nervosa [6,10]. É comum ocorrer recidiva local após a cirurgia.

O prognóstico de pacientes com tumores intradurais-extramedulares é reservado [3,6,11], estimase recidiva de até $72 \%$ dos casos após a excisão cirúrgica [12].

O trabalho tem como objetivo descrever um caso de hemiparesia nos membros torácico e pélvico do lado direito em cão, ocasionada por compressão da medula espinhal devido à neoplasia intradural-extramedular classificada como Schwanoma.

\section{RELATO DO CASO}

Foi atendido no Serviço de Neurologia do Hospital de Clínicas Veterinárias (HCV), Faculdade de Veterinária, Universidade Federal do Rio Grande do Sul (UFRGS), um cão, Pastor Belga, macho, 8 anos, o proprietário relatava que o animal apresentava episódios de dor e extensão do pescoço há mais de um ano, arrastava e tinha falta de força no membro torácico direito. $\mathrm{O}$ exame neurológico evidenciou hiperestesia à palpação da articulação atlanto-occipital, déficit de reação postural e hemiparesia nos membros torácico e pélvico do lado direito. O exame radiográfico simples da região cervical cranial não demonstrou alterações. $\mathrm{O}$ exame mielográfico evidenciou existência de massa sub-dural extramedular no lado direito junto à articulação atlanto-occipital, sendo provavelmente uma neoplasia. Assim, optou-se pela técnica cirúrgica de hemilaminectomia dorsolateral cervical cranial. Foi realizada abordagem cirúrgica às vértebras através de incisão de pele na linha média dorsal, secção de tecido adiposo e fáscia subcutâneas desde a protuberância do occipital até o processo espinhoso dorsal de C4. A dissecção prosseguiu na linha média desde a protuberância do occipital, dorsal ao arco de $\mathrm{C} 1$ (atlas) e processo espinhoso de $\mathrm{C} 2$ (áxis). Os músculos foram rebatidos com um elevador periosteal e o afastamento lateral foi mantido por afastadores auto-estáticos de Weitlander. Posteriormente foi feita a remoção parcial da lâmina dorsolateral do atlas (C1) para permitir o acesso ao cordão espinhal confirmando-se a existência de massa tumoral a qual foi parcialmente removida. O fechamento do ferimento cirúrgico foi realizado por meio de sutura contínua simples dos músculos na rafe tendínea em suas camadas respectivas com fio absorvível (Vicryl 2-0), rafia do tecido subcutâneo com padrão de sutura contínua simples com fio absorvível (Vicryl 2-0) e para pele padrão de sutura isolado simples com fio não absorvível (mononáilon 2-0). O material removido foi enviado para exame histológico. Ao exame histológico foi observada a presença de células com citoplasma vacuolizado e células alongadas e fusiformes arranjadas em redemoinhos, sendo compatível com Schwanoma. Na avaliação neurológica realizada no dia seguinte ao da cirurgia verificou-se agravamento do quadro clínico, instalação de tetraparesia associada à ataxia e Síndrome de Horner (ptose palpebral, miose, enoftalmia e projeção da $3^{\mathrm{a}}$ pálpebra) no lado direito. Trinta dias após a cirurgia, verificou-se discreta hemiparesia no lado direito, com regressão dos demais sinais, permitindo caracterizar uma melhora em relação ao quadro précirúrgico. Dois meses e meio após a cirurgia, o proprietário trouxe o cão alegando que, havia duas semanas, o mesmo começara a piorar, manifestando dor ao realizar movimentos com a cabeça. Foi feita uma nova mielografia que evidenciou aumento do tumor em relação ao tamanho inicial. Foi realizado um novo procedimento cirúrgico com ressecção do tumor, porém, ao término da intervenção, o animal foi a óbito. 

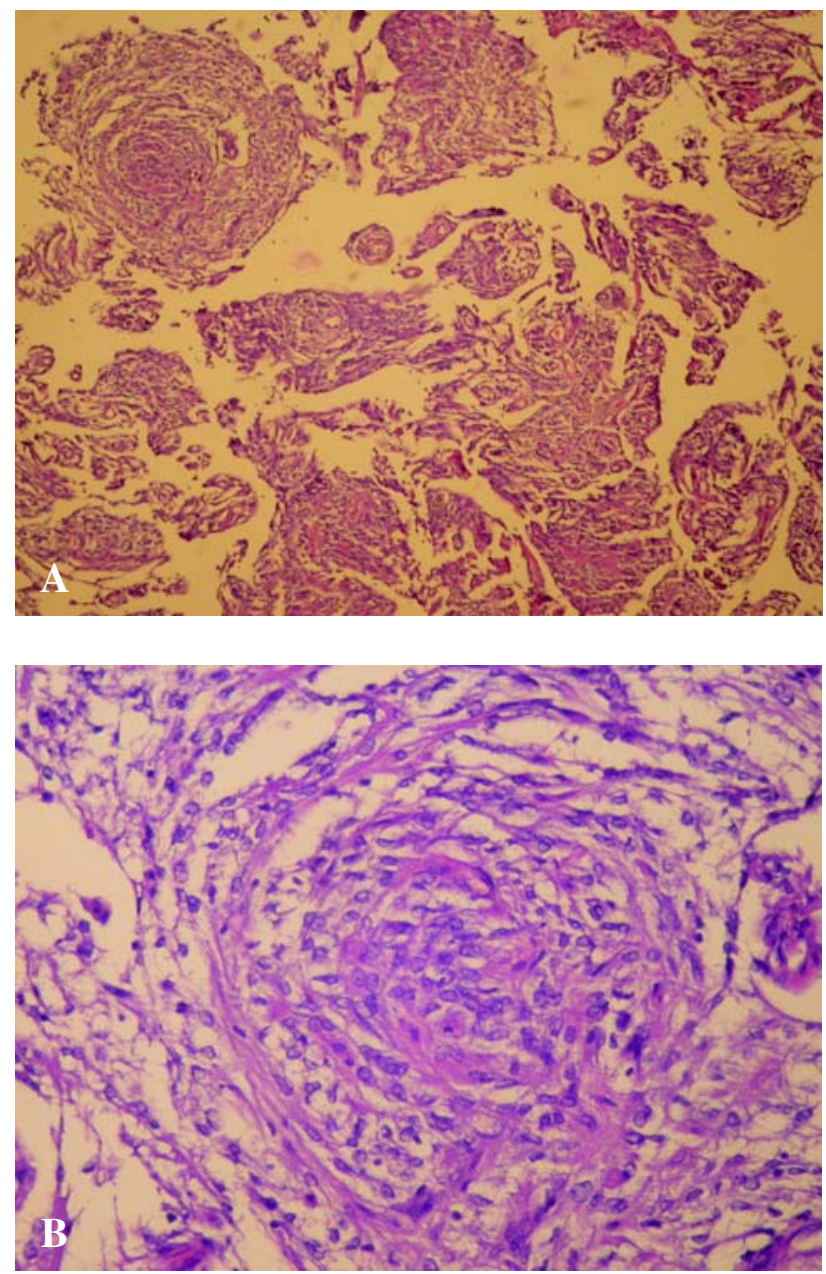

Figuras 1A e 1B. Células com citoplasma vacuolizado, células alongadas e fusiformes arranjadas em redemoinhos. Moderado tecido conjuntivo.

\section{DISCUSSÃO}

Neste caso, a compressão da medula espinhal ocorreu devido ao desenvolvimento de tumor subduralextramedular diagnosticado como Schwanoma conforme demonstraram os exames mielográfico e histológico, respectivamente. A descompressão produzida pela hemilaminectomia dorsolateral foi eficiente, num primeiro momento, como forma de tratamento, o que ficou evidente pela redução da gravidade dos sinais neurológicos.

No entanto, aproximadamente 75 dias após a cirurgia constatou-se agravamento dos sinais. $\mathrm{O}$ exame mielográfico evidenciou aumento de volume do tumor em relação ao inicial. Foi realizada exérese do tumor, porém ao término do procedimento cirúrgico o paciente foi a óbito. Tal fato condiz com a literatura, onde se relata que apenas uma pequena porcentagem desses tumores é completamente removida e as recidivas são altas.

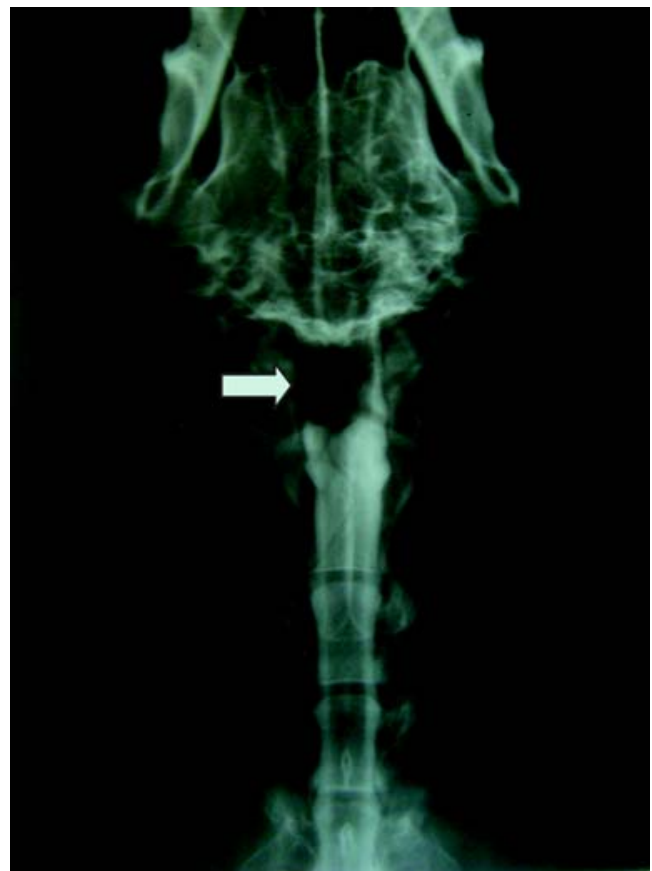

Figura 2. Exame mielográfico na posição ventrodorsal com imagem característica de compressão subdural-extramedular junto à articulação atlanto-occipital.

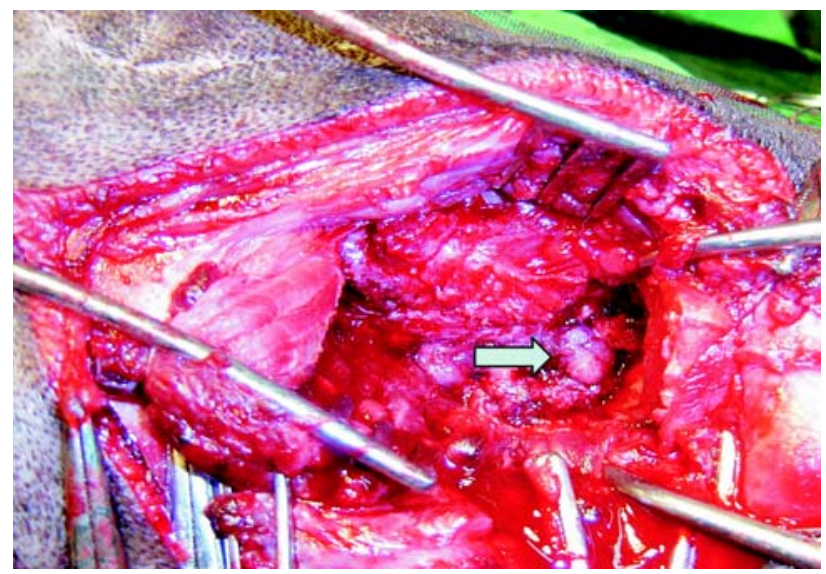

Figura 3. Visualização parcial do tumor.

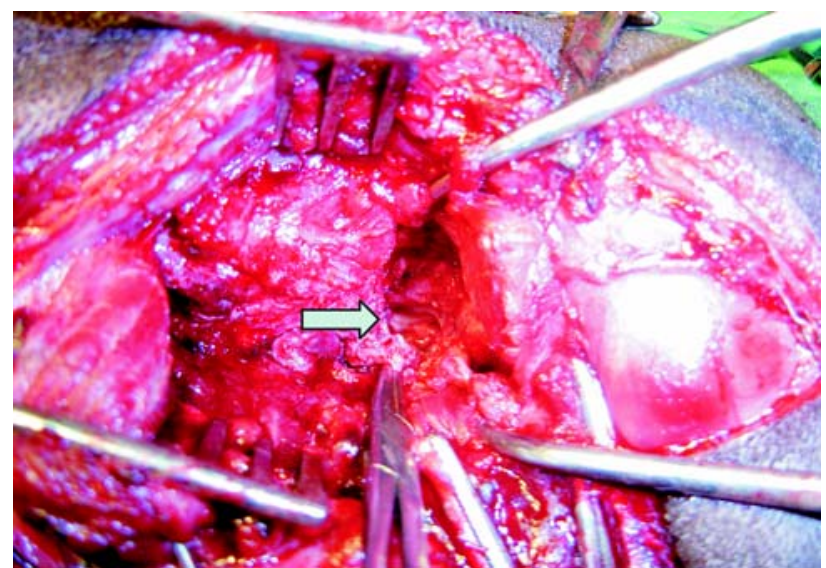

Figura 4. Visualização da medula espinhal livre após remoção parcial do schwanoma. 


\section{REFERÊNCIAS}

1 Bagley R.S., Kornegay J. N., Page R.L. \& Thrall D.E. 1998. Sistema Nervoso Central. In: Manual de Cirurgia de Pequenos Animais. 2 ed. São Paulo: Roca, pp. 2546-2548.

2 Bailey C.S. 1990. Long-term survival after surgical excision of a schwanoma of the sixth cervical spinal nerve in a dog. Journal of the American Veterinary Medical Association.196: 754-756.

3 Braund K.G. 1986. Neurological Diseases. In: Clinical Syndromes in Veterinary Neurology. Baltimore: Williams \& Wilkins, pp.141-146.

4 Chrisman C.L. 1985. Paresia ou Paralisia de um Membro. In: Neurologia dos Pequenos Animais. São Paulo: Roca, p.385.

5 Fenner W.R. 1997. Moléstias do Cérebro. In: Medicina Interna Veterinária. São Paulo: Manole, pp.955-960.

6 Fossum T.W. 2002. Cirurgia da Espinha Cervical. In: Cirurgia de Pequenos Animais. São Paulo: Roca, pp.1211-1215.

7 Jones T.C. \& Koestner A. 2000. Sistema Nervoso. In: Patologia Veterinária. São Paulo: Manole, pp.1317-1319.

8 Kornegay J.N. 1998. Patogênese das Afecções do Sistema Nervoso Central. In: Manual de cirurgia de Pequenos Animais. 2 ed. São Paulo: Roca, p.1246.

9 Morris J. \& Dobson J. 2001. Nervous System. In: Small Animal Oncology. Oxford: Blackwell Science Ltd, p.200.

10 Salisbury S.K. 1998. Surgery for Oncologic Emergencies. In: Cancer in Dogs and Cats. USA: Williams \& Wilkins, pp.258-264.

11 Theilen G.H. \& Madewell B.R. 1979. Tumors of the Skin and Subcutaneous Tissues. In: Veterinary Cancer Medicine. Philadelphia: Lea \& Febiger, p.164.

12 Tilley L.P. \& Smith F.W.K. 2003. Consulta Veterinária em 5 minutos. 2.ed. São Paulo: Manole, p.1186.

13 Wheeler S.J \& Sharp J.H. 1999. Diagnóstico e Diagnóstico Diferencial. In: Diagnóstico e Tratamento Cirúrgico das Afecções Espinais do Cão e do Gato. São Paulo: Manole, p.31. 